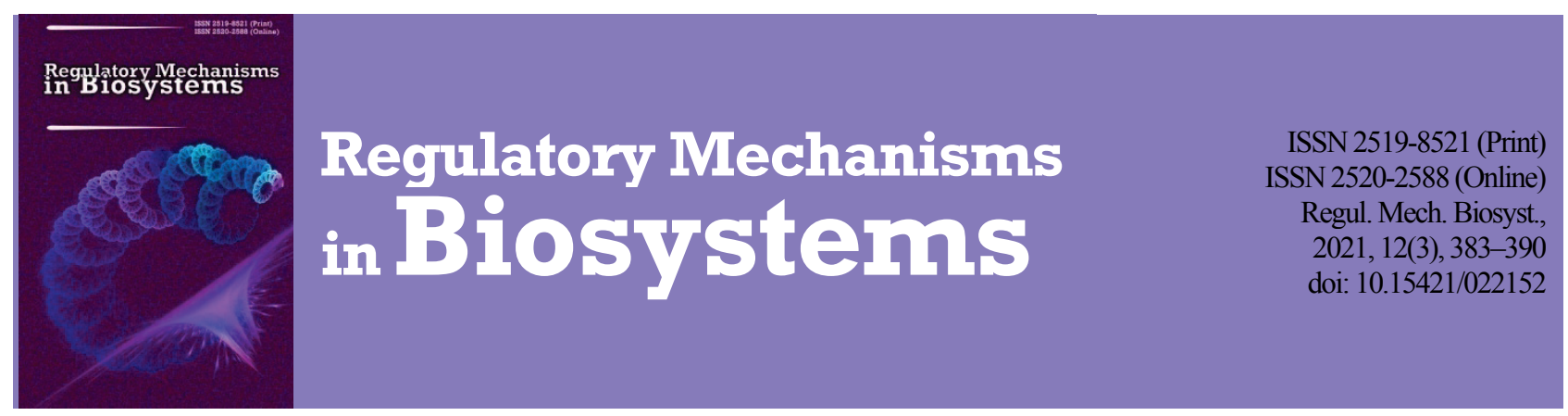

\title{
Influence of metal nanocarboxylates and different water supply conditions on efficiency of soybean-rhizobial symbiotic systems
}

\author{
S. Y. Kots, L. I. Rybachenko, T. P. Mamenko, K. P. Kukol, P. P. Pukhtaievych, O. R. Rybachenko \\ Institute of Plant Physiology and Genetics, National Academy of Sciences of Ukraine, Kyiv, Ukraine
}

Article info

Received 10.07.2021

Received in revised form 07.08 .2021

Accepted 08.08.202

Institute of Plant

Physiology and Genetics,

National Academy

of Sciences of Ukraine,

Vasylkivska st., 31/17,

Kyiv, 03022, Ukraine

Tel.: +38-096-125-16-76.

E-mail:veselika@ukr.net

Kots, S. Y., Rybachenko, L. I., Mamenko, T. P., Kukol, K. P., Pukhtaievych, P. P., \& Rybachenko, O. R. (2021). Influence of metal nanocarboxylates and different water supply conditions on efficiency of soybean-rhizobial symbiotic systems. Regulatory Mechanisms in Biosystems, 12(3), 383-390. doi:10.15421/022152

Insufficient water supply is one of the main factors that significantly reduce the activity of nitrogen fixation by legume-rhizobial symbiotic systems. That is why comprehensive research on aspects of their resistance to water stress and the search for scientifically substantiated ways to improve the existing ones and develop modern, competitive technologies of growing legumes in arid conditions are becoming especially relevant. The aim of the work was to investigate the processes of formation and functioning of soybean-rhizobial symbiotic systems developed under conditions of different water supply and influence of nanocarboxylates of cobalt, ferum, germanium, chromium, cuprum and molybdenum. The nanoparticles of specified metal nanocarboxylates were used as components of the inoculation suspension of rhizobia of Tn5 mutant B1-20 for soybean seed treatment. A model drought lasting 14 days was created by controlled irrigation. Microbiological and physiological research methods were used in the study. We determined that insufficient water supply caused a significant decrease in the nodulation potential of rhizobia and the intensity of molecular nitrogen fixation by symbiotic systems formed with the participation of soybean plants and nodule bacteria without adding these metal nanocarboxylates to the inoculation suspension. Application of most of the metal nanocarboxylates used as components of the inoculation suspension mitigated the negative impact of stress on the investigated parameters. The study revealed the stimulating effect of cobaltnanocarboxylate on the activity of molecular nitrogen fixation, which was more pronounced in the conditions of insufficient water supply. Symbiotic soybean systems formed with the participation of nodule bacteria containing germaniumcarboxylate nanoparticles were proved to be the least sensitive to the negative impact of insufficient water supply. This was indicated by high rates of nodulation and nitrogen-fixing activity compared with other studied symbiotic systems. We confirmed that the addition of chromium nanocarboxylate to the inoculation suspension of rhizobia provided the highest rates of nodulation and nitrogen-fixing activity of soybean root nodules under optimal growing conditions and, at the same time, had no noticeable positive effect under water stress. We determined that cuprum and molybdenum nanocarboxylates, as components of the inoculation suspension, regardless of the water supply level, had a less notable positive effect on the processes of nodule formation and nitrogen fixation, and in some cases even led to a decrease in the investigated values for control plants. Thus, the study demonstrated that the use of germanium, cobalt and ferum nanocarboxylates as components of the bacterial suspension helped to increase the adaptation of the formed legume-rhizobial symbiotic systems to water stress, as evidenced by the maximum indexes of nodulation and molecular nitrogen fixation in the context of insufficient water supply and recovery of their level to optimal after the stress influence had ended. Based on the results, it was concluded that inoculation of seeds by the complex bacterial preparations made on the basis of Bradyrhizobium japonicum B1-20 with a content of germanium, cobalt and ferum nanocarboxylates in the concentration of 1:1000 can become one of the important means in soybean growing technologies of increasing the nitrogen-fixing potential and resistance of plants to insufficient water supply.

Keywords: Bradyrhizobium japonicum; soybean; nanoparticles of chelated metals; fixation of molecular nitrogen; nodulating rhizobia activity.

\section{Introduction}

An important factor in restoring and preserving soil fertility, maintainning the greening of agricultural production and saving resources is the maximum realization of the potential of plant-microbial interactions. Symbiosis of legumes with nitrogen-fixing nodule bacteria and its use is one of many examples of intensification of agro-industrial production (Wang et al., 2018). The use of microbial preparations made on the basis of active competitive strains of rhizobia in the technologies of growing crops provides optimal conditions for plant nutrition with available sources of nitrogen and prevents its unproductive losses due to mineralization and subsequent leaching from the soil, and also prevents nitrate pollution of groundwater, which allows obtaining higher yields of ecologically safe crop products (Sonali et al., 2020).

However, the process of molecular nitrogen fixation by symbiotic systems, as well as the yield and protein productivity of soybeans, largely depend on environmental conditions. Drought is a complex integral factor that leads to pathological changes at different levels of symbiosis - from subcellular to organismic. Lack of water can indirectly affect microorganisms, in particular through the plant, by inhibiting its growth and development, reducing the amount of exudate released by it into the rhizosphere, as well as directly on the rhizobia by impairing the diffusion processes and changing the nutrient concentrations (Kunert et al., 2016; Kibido et al., 2019). In the conditions of excessive moisture in the soil, the respiratory function of the nodule bacteria becomes impaired, leading to decrease in their number in the ecotope. It was demonstrated that under the influence of insufficient water supply, nitrogen fixing activity of soybeans may decrease to 26\% and more (Aldasoro et al., 2019; Schwember et al., 2019). It is known that the degree of inhibition of the formation and functioning of symbiosis in the absence of moisture depends on the duration and intensity of the stress factor. For example, moderate water stress is the cause of reduction of the number of root nodules, while prolonged exposure not only reduces their number, but also their size. The negative effect of drought on the processes of nodule formation and their nitrogen- 
fixing activity also depends on the stage of plant growth. It was found that the decrease in water supply during the vegetative growth of legumes had more harmful effects on the development and functioning of nodules than in the reproductive period (Parvaze et al., 2018). Prolonged water deficiency causes a number of morphological changes in rhizobia, which ultimately lead to worsening of process of infection of the legume. In addition, decrease in soil moisture from $50 \%$ to $30 \%$ of total moisture content significantly reduced the number of infectious filaments formed inside the root hairs, and completely inhibited the formation of nodules in Trifolium subterraneum (Worrall \& Roughley, 1976). Similar results were obtained on Vicia faba plants by modeling water deficiency with polyethylene glycol (Zahran \& Sprent, 1986). Inhibition of nitrogen fixation by legumes led to a significant decrease in seed productivity of these crops in many arid and semi-arid regions of the world.

Today, the introduction of nanotechnologies into crop production, which are now characterized by the most dynamic development in the agro-industrial complex, can make a significant contribution to increasing the adaptability of cultivated plants, and hence symbiotic systems, to adverse ecological factors.The application of nanotechnological developments in agriculture facilitates an increase in the qualitative and quantitative indicators of the obtained products (Solanki et al., 2015) and at the same time reduces environmental pollution by agrochemicals (Prasad et al., 2017).

The use of biologically active nano additives, in which microelements are used as plant growth stimulators and activators of metabolic processes, is promising. Metal salts in these preparations, used for the treatment of plants and seeds, have been replaced by more accessible forms of microelements, which provide minimum requirements to their concentrations and reduce environmental pollution (Du et al., 2017). At present, it has been proven that microelements affect the direction of biochemical reactions in the plant body through the impact on biocolloids. They are involved in a number of redox processes in plants and affect the synthesis of proteins, fats, carbohydrates, as well as being a component of vitamins, hormones and other biologically active substances. They are also able to indirectly influence the processes that increase the resistance of plants to adverse environmental factors (Dimkpa et al., 2017).

Nanoparticles have increased reactivity and efficiency. Their use in crop production, in particular, as microfertilizers, helps to increase the resistance to adverse weather conditions and increase yields (on average by 1.5-2.0 times) of almost all food crops (potatoes, cereals, vegetables, fruits and berries) and industrial crops (cotton, flax, etc.) (Dimkpa et al., 2017). Laboratory studies shown that crops grown from seeds coated by nanomaterials such as nanosilver have increased water absorption ability (Patra \& Baek, 2017). Other studies found that the seeds treated with nanoparticles exceeded the control variant by $73 \%$ according to the parameters of dry weight and were characterized by three times higher content of vitamins (Javaid et al., 2020). Moreover, plants grown from nanoparticletreated seeds had 90\% higher drought resistance (Rahimi et al., 2016). Timely assimilation of minerals, especially in stages of growth and development of plants to which moisture deficiency is critical, may affect the adaptive changes in metabolism and increase their drought resistance, as well as reduce yield losses.

Therefore, study of the formation and functioning of soybean-rhizobial systems, as well as the search for ways to influence the realization of their nitrogen-fixing potential under stressful drought conditions has considerable practical interest. Therefore, we studied the possibility of using nanocarboxylates of cobalt, ferum, germanium, chromium, cuprum and molybdenum metals as components of the inoculation suspension $\mathrm{Bra}$ dyrhizobium japonicum for soybean seed treatment to increase the efficiency of nodulation and fixation of molecular nitrogen by root nodules of soybean in the ontogenesis stage when lack of moisture is critical and after resuming watering the plants.

\section{Materials and methods}

The objects of the study were symbiotic systems, formed with participation of soybean (Glycine max (L.) Merr.) Almaz variety and the nodule bacteria Bradyrhizobium japonicum B1-20 by adding to the medium their cultivation of chelated metals: cobalt, ferum, germanium, chromium, cuprum and molybdenum. In the work we used the nodule bacteria from the collection of $\mathrm{N}_{2}$-fixing microorganisms of the Institute of Plant Physiology and Genetics NAS of Ukraine. The nodule bacteria were grown in test tubes on yeast-mannitol agar at the temperature of $28^{\circ} \mathrm{C}$ for 7 days. To prepare a liquid suspension of bacteria, they were washed from the surface of the agar medium and inoculated into Erlenmeyer flasks ( $200 \mathrm{~mL}$ capacity) with liquid medium, that in corresponding variants contained chelated metals in the ratio of $1: 1,000$. The inoculum was added to the flasks in the concentration of $2 \%$ of volume of the nutrient medium. Cultivation of freshly prepared suspension of rhizobia containing nanometals was carried out for 6 days in the temperature of $+26-28^{\circ} \mathrm{C}$ on a rotary shaker with the speed of $220 \mathrm{rpm}$, which provided constant aeration of the growing medium. The titer of bacteria was $10^{8}$ cells $/ \mathrm{mL}$.

The micronutrients we used were provided by Avatar LLC Research and Production Company (Kyiv, Ukraine). They are obtained in two stages: 1 - obtaining an aqueous colloidal solution of nanoparticles of microelements by dispersing highly purified granules of the corresponding metals by impulses of electric current in deionized water; 2 - obtaining metal carboxylates by the reaction of direct interaction of the obtained nanoparticles with food carboxylic acid.

The research was performed in the controlled conditions of the vegetation experiment. Prior to inoculation, soybean seeds were sterilized with $70 \%$ ethanol solution and rinsed with running water, then inoculated with a suspension of rhizobia containing these nanometals. The scheme of the experiment included the following variants:

1) seeds + B. japonicum B1-20 (60\% of total moisture content (control 1);

2) seeds $+($ B. japonicum B1-20 + cobalt nanocarboxylate) $(60 \%$ of total moisture content);

3) seeds + (B. japonicum B1-20 + ferum nanocarboxylate) $(60 \%$ of total moisture content);

4) seeds + (B. japonicum B1-20 + germanium nanocarboxylate) (60\% of total moisture content);

5) seeds $+($ B. japonicum B1-20 + chromium nanocarboxylate $)(60 \%$ of total moisture content);

6) seeds + (B. japonicum B1-20 + cuprum nanocarboxylate) $(60 \%$ of total moisture content);

7) seeds + (B. japonicum B1-20 + molybdenum nanocarboxylate) (60\% of total moisture content);

8) seeds + B. japonicum B1-20 (30\% of total moisture content) (control 2);

9) seeds + (B. japonicum B1-20 + cobalt nanocarboxylate) $(30 \%$ of total moisture content);

10) seeds $+($ B. japonicum B1-20 + ferum nanocarboxylate) $(30 \%$ of total moisture content);

11) seeds + (B. japonicum B1-20 + germanium nanocarboxylate) (30\% of total moisture content);

12) seeds + (B. japonicum B1-20 + chromium nanocarboxylate) ( $30 \%$ of total moisture content);

13) seeds + (B. japonicum B1-20 + cuprum nanocarboxylate) $(30 \%$ of total moisturecontent);

14) seeds + (B. japonicum B1-20 + molybdenum nanocarboxylate) (30\% of total moisture content).

The plants were grown in $4 \mathrm{~kg}$ pots at natural light and temperature, at optimal (60\% of total moisture content) and insufficient (30\% of total moisture content) water supplies. The substrate for growing was river sand. The source of mineral nutrition was a nutrient mixture of Helrigelwith 0.25 of nitrogen norm. The humidity of the substrate was maintained by controlled watering. Drought was created for two weeks (from the stage of three true leaves to blossom), after which watering was restored to $60 \%$ of TMC in the stage of beans' formation.

For the research, the plants were selected in three stages - of the budding (finishing of the first week of drought), of the blossom (finishing of the second week of drought) and of the beans formation (resumption of watering).

The nodulation ability of $B$. japonicum was determined according to the number and weight of root nodules. Nitrogen-fixing activity (NFA) (acetylene reduction activity) was measured on a gas chromatograph Agilent GC system 6850 (USA) with a flame-ionization detector (Hardy, 
1968). The separation of gases was carried out on a column (Supelco Porapak N) at a thermostat temperature of $55^{\circ} \mathrm{C}$ and a detector $-150^{\circ} \mathrm{C}$. The carrier gas was helium $(20 \mathrm{~mL}$ per 1 minute). The volume of the analyzed sample gas mixture was $1 \mathrm{~cm}^{3}$. As a standard, pure ethylene (Sigma-Aldrich, No 536164 USA) was used.

The tables and figures show the arithmetic mean values and their standard errors $(x \pm \mathrm{SE})$. The reliability of the differences between the samples was evaluated using the single-factor dispersion analysis (ANOVA), using the Mann-Whitney U-criterion. Differences were considered to be significant at $\mathrm{P}<0.05$.

\section{Results}

Analysis of the number and weight of root nodules in symbiotic systems of soybean-B. japonicum B1-20 revealed the following: against the background of optimal water supply for plants inoculated with a biological preparation containing cobalt nanocarboxylate, there was an increase in the number of root nodules compared with the plants of control variant 1 by $108 \%$ and $94 \%$ - in the blossom stage and in the beans formation stage, respectively (Table 1, 2).

\section{Table 1}

Number of nodules (pcs.plant) on the roots of soybean plants when using metal nanocarboxylates in different water supply conditions $(x \pm S E, n=8)$

\begin{tabular}{|c|c|c|c|}
\hline \multirow{2}{*}{ Variants } & \multicolumn{3}{|c|}{ Stages of development in plants } \\
\hline & budding & blossom & beans formation \\
\hline \multicolumn{4}{|c|}{ Optimal water supply (60\% of total moisturecontent) } \\
\hline B. japonicum B1-20 (control 1) & $15.1 \pm 1.1$ & $14.5 \pm 1.1$ & $15.3 \pm 0.5$ \\
\hline Cobalt nanocarboxylate & $59.1 \pm 3.1 * *$ & $30.2 \pm 2.1 *$ & $29.7 \pm 2.1 *$ \\
\hline Ferum nanocarboxylate & $30.1 \pm 1.1^{\times}$ & $28.2 \pm 3.1^{\times}$ & $50.1 \pm 4.3^{\times}$ \\
\hline Germanium nanocarboxylate & $34.7 \pm 3.1^{\neq}$ & $46.7 \pm 2.2^{\#}$ & $22.1 \pm 1.9^{\neq}$ \\
\hline hromium nanocarboxylate & $21.7 \pm 1.8^{\prime \prime}$ & $24.2 \pm 1.1^{\prime \prime}$ & $33.5 \pm 1.2^{巛 \ll}$ \\
\hline & $17.3 \pm 1.6$ & $36.7 \pm$ & $31.7 \pm 2.2^{\prime \prime \prime \prime}$ \\
\hline Molybdenum nanocarboxylate & $14.7 \pm 1.5$ & $36.1 \pm 1.9^{++}$ & $34.3 \pm 1.1^{++}$ \\
\hline \multicolumn{4}{|c|}{ Insufficient water supply (30\% of total moisturecontent) } \\
\hline B. japonicum B1-20 (control 2) & $8.3 \pm 0.9$ & $13.1 \pm 1.3$ & $19.6 \pm 1.2$ \\
\hline Cobalt nanocarboxylate & $20.1 \pm 2.1 * *$ & $16.7 \pm 2.2$ & $25.2 \pm 3.1 * *$ \\
\hline lanocarboxylate & $15.3 \pm 1.3^{\times}$ & $19.7 \pm 1.7$ & $27.3 \pm 1.9^{\times x}$ \\
\hline Germanium nanocarboxylate & $32.1 \pm 4.5^{\#}$ & $38.1 \pm 2.1^{\#}$ & $20.3 \pm 1.1$ \\
\hline Chromium nanocarboxylate & $12.1 \pm 1.1$ & $10.5 \pm 1.9$ & $28.2 \pm 2.1^{\prime \prime}$ \\
\hline Cuprum nanocarboxylate & & $24.7 \pm 1.3^{\prime \prime}$ & $18.2 \pm 0.8$ \\
\hline Molybdenum nanocarboxylate & $16.7 \pm 1.9^{+}$ & $17.1 \pm 0.9$ & $34.1 \pm 1.6^{++}$ \\
\hline
\end{tabular}

The weight of nodules was at the level of control plants 1 . The exception was the budding stage, where increases in both the number and weight of root nodules were observed compared to those of the control by $293 \%$ and $133 \%$, respectively. In the conditions of insufficient watering, the use of cobalt in the suspension culture provided $140 \%$ and $39 \%$ increases in the number and weight of nodules in the budding stage (Fig. 1), and $28 \%$ and $118 \%$ in the beans formation stage respectively. During the blossom period, on the roots of plants of this variant, an increase of only the number of nodules was recorded, equaling $29 \%$ compared with control plants 2.

The use of ferum nanocarboxylate as a component of the rhizobia inoculation suspension also led to an increase in the number of root nodules in the conditions of optimal water supply by $100 \%, 95 \%$ and $226 \%$ in the budding, blossom and beans formation stages compared with the plants of control 1 (Table 1). At the same time, their weight increased compared with the plants of the control in the budding and blossom stages by $57 \%$ and $41 \%$, respectively (Table 2 ). Insufficient water supply negatively affected the formation of symbiotic systems, however, ferum nanocarboxylate caused a fairly high rate of nodulation activity against control plants 2 , and that is, an increase in the number and weight of root nodules by $84 \%$ and $50 \%$ - in the budding stage (Fig. 1), by $52 \%$ and $176 \%$ in the blossom stage and by $39 \%$ and $37 \%$ in the beans formation stage.

The study of the effect of germanium nanocarboxylate in the microbial preparation on the symbiotic systems of soybeans revealed that in optimal plant growing conditions this element became the cause of increase in the number of root nodules compared with the control variant 1 by $131 \%$ in the budding stage, by $222 \%$ in the blossom stage and by $44 \%$ in the beans formation stage, and also promoted increase in their weight by $88 \%, 68 \%$ in the stages of budding and blossom, respectively (Tables 1 and 2). In the budding stage, on the roots of plants inoculated using biological preparation with germanium nanocarboxylate, which were under stress for 7 days, the number of root nodules exceeded control 2 by $284 \%$ (Fig. 1), and was at the level of plants of the same variant, grown at the optimal level of water supply. At the same time, their weight, being at the level of the control variant 2 , decreased by $40 \%$ compared with the plants of the same variant grown with optimal water supply. The two-week effect of the stress factor caused decrease in the number and weight of root nodules in this variant compared to the same plants grown in the conditions of $60 \%$ of complete moisture content. At the same time, compared with plants of control 2, the examined indicators were higher by 192 and $138 \%$, respectively. In the beans formation stage, the number of root nodules in plants inoculated using rhizobia with germanium nanocarboxylate was lower than in the previous development phases and slightly exceeded the control 2 . At the same time, we recorded $43 \%$ increase in the weight of nodules compared with the plants of the same control. A similar effect of germanium in the content of inoculant was found in plants grown in optimal conditions of water supply (Table 1,2).

Table 2

Dry weight of root nodules (mg of dry substance/plant) of soybean plants using metal nanocarboxylates in different conditions of water supply $(\mathrm{x} \pm \mathrm{SE}, \mathrm{n}=8)$

\begin{tabular}{|c|c|c|c|}
\hline \multirow{2}{*}{ Variants } & \multicolumn{3}{|c|}{ Stages of development in plants } \\
\hline & budding & blossom & beans formation \\
\hline \multicolumn{4}{|c|}{ Optimal water supply ( $60 \%$ of total moisturecontent) } \\
\hline B. japonicum B1-20 (control 1) & $51.1 \pm 8.1$ & $82.1 \pm 8.2$ & $117.4 \pm 16.2$ \\
\hline Cobalt nanocarboxylate & $119.2 \pm 11.2 * *$ & $82.2 \pm$ & $143.1 \pm 14.3$ \\
\hline Ferum nanocarboxy & $81.2 \pm 7.1^{\times}$ & $116.1 \pm 6.2^{\times x}$ & $126.3 \pm 12.1$ \\
\hline & $96.3 \pm 5.3^{ \pm}$ & $138.3=$ & \pm 12.2 \\
\hline & $109.1 \pm 6.2^{211}$ & $123.2 \pm$ & $271.2 \pm 14.1^{\text {'II }}$ \\
\hline Cuprun & $95.3 \pm 2.4 "$ & 141.1 & $88.1 \pm 7.3 "$ \\
\hline Molybdenum nanocarboxylate & $51.2 \pm 4.3$ & $174.1 \pm 10.1^{++}$ & $89.1 \pm 6.4^{+}$ \\
\hline \multicolumn{4}{|c|}{ Insufficient water supply ( $30 \%$ of total moisturecontent) } \\
\hline B. japonicum $\mathrm{B} 1-20$ (control 2) & $56.1 \pm 7.1$ & $37.2 \pm 4.2$ & \\
\hline Cobalt nanocarboxylate & $78.2 \pm 8.2^{*}$ & $41.1 \pm 3.3$ & $107.2 \pm 13.2 * *$ \\
\hline Ferum nanocarboxylate & $84.2 \pm 5.3^{\times}$ & $102.4 \pm 9.7^{\times \times x}$ & $67.3 \pm 5.1^{\times}$ \\
\hline Germanium nanocarboxylate & $59.1 \pm 4.3$ & $88.1 \pm 9.1^{\#}$ & $71.4 \pm 6.1^{\neq}$ \\
\hline Chromium nanocarboxylate & $29.3 \pm 3.1^{\prime \prime}$ & $37.2 \pm 5.4$ & $61.3 \pm 5.2^{\prime \prime}$ \\
\hline Cuprum nanocarboxylate & $37.3 \pm 2.4 "$ & $81.4 \pm 8.3^{\prime \prime \prime \prime}$ & $66.2 \pm 7.1^{\prime \prime}$ \\
\hline Molybdenum nanocarboxylate & $44.2 \pm 6.2$ & $35.3 \pm 2.4$ & $79.4 \pm 5.8^{+}$ \\
\hline
\end{tabular}

Note: see Table 1.

The use of chromium nanocarboxylate as a component of the inoculation suspension led to an increase number of root nodules by $44 \%, 67 \%$, $118 \%$ and the weight - by $114 \%, 50 \%, 132 \%$, compared with the control 1 in the stage of budding, blossom and beans formation, respectively. In the conditions of insufficient water supply, the positive effect of the use of chromium was less pronounced, in particular during the budding stage this element provided $44 \%$ increase in the number of nodules while reducing their weight by $48 \%$ compared with control 2 . During the period of resumption of watering, we recorded $44 \%$ and $24 \%$ increases in the number and weight of nodules in this variant respectively (Tables 1 and 2).

Against the background of optimal water supply in the budding stage, plants inoculated using a biological preparation containing cuprum nanocarboxylate were characterized by $86 \%$ increase in only the weight of nodules, while in the blossom stage $153 \%$ and $72 \%$ increases in both the number and weight of nodules were recorded compared with the control plants 1 . In the stage of beans formation, when the number of root nodules increased by $107 \%$, their weight decreased by $25 \%$ compared with the plants of corresponding control.

After a week-long insufficient water supply (budding stage), the symbiotic systems formed by rhizobia with copper nanocarboxylate exceeded control 2 by $35 \%$ by the number of nodules, whereas the nodules weight was by $34 \%$ lower than the same control. In the blossom stage ( 14 days of drought), the plants of this variant were characterized by an increase in 
both the number and weight of nodules compared with control 2 by $90 \%$ and $116 \%$ respectively. In the period of the resumption of watering (beans formation stage), having the number of nodules at the level of control variant 2 , we recorded $35 \%$ increase in their weight (Tables 1 and 2 ).

Evaluation of nodulation activity in symbiotic systems which were developed based on bacterial preparation with the addition of molybdenum nanocarboxylate showed that in the optimal conditions of soybean cultivation, this element caused no significant changes in the number and weight of root nodules compared with control 1 . The exception was the blossom stage, where the number of nodules increased by $148 \%$ compared with the same control, as well as the stage of beans formation, where with the increase in the number of nodules (by 124\%) there was a decrease in their weight (by $25 \%$ ). In the conditions of the influence of the stress factor, the number of nodules occurred in all investigated stages of plant development increased from $31 \%$ to $100 \%$. At the same time, in the budding stage the weight of nodules decreased by $22 \%$ compared with control 2, and in the stage of beans formation this indicator increased by $61 \%$ compared with the same control (Tables 1 and 2 ).
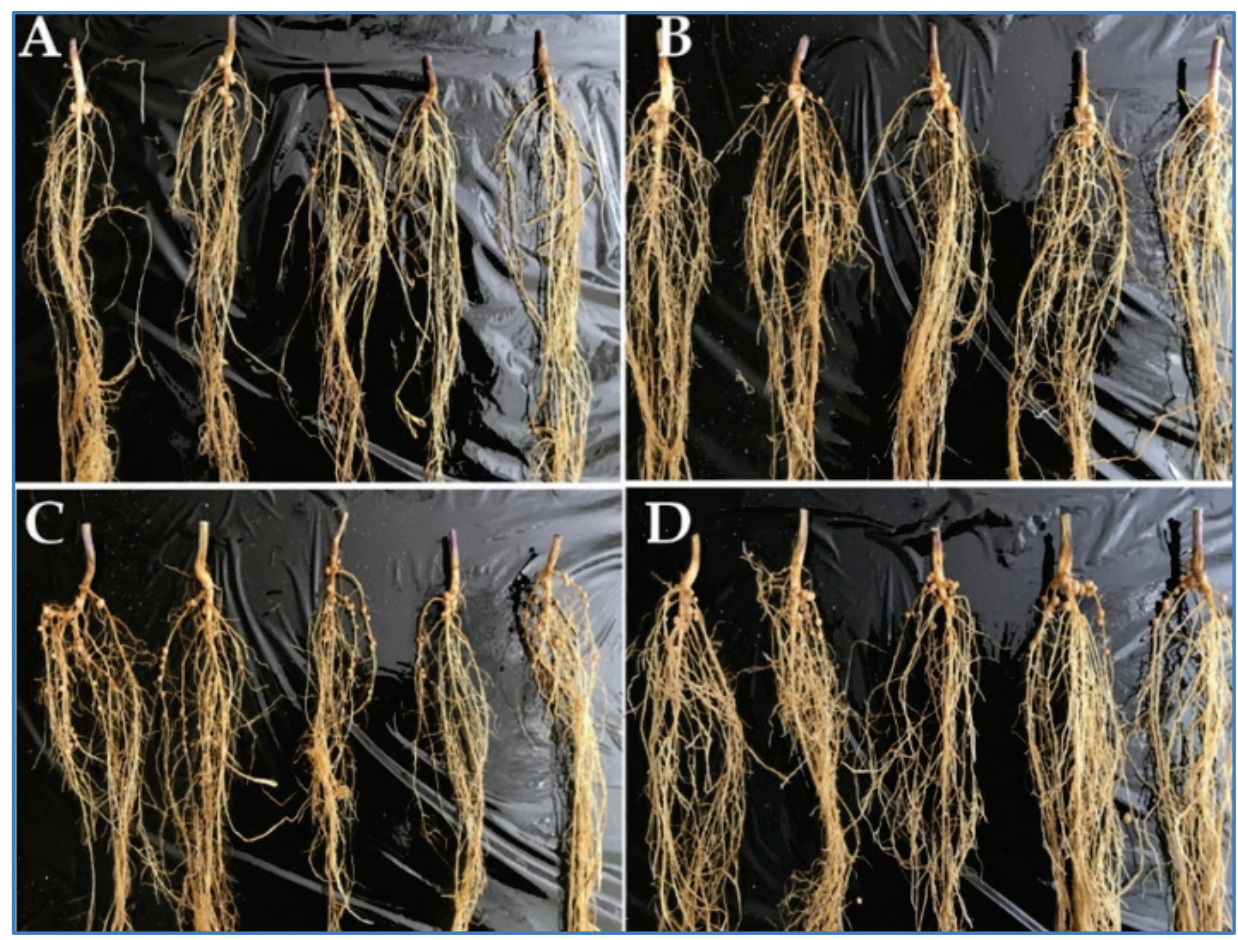

Fig. 1. The number of nodules on the roots of soybean: $A$ - control $30 \%, B$ - cobalt $30 \%, C$ - germanium $30 \%, D$ - ferum, $30 \%$ (the budding stage)

The study of nitrogen-fixing activity of the symbiotic systems revealed that the action of one- and two-week stress (budding and blossom stages) caused plants inoculated using biopreparation with cobalt nanocarboxylate to exceed control 2 by $23 \%$ and $52 \%$ respectively, and in the period of watering (beans formation stage) - by $157 \%$. At the optimal water supply, the nitrogen-fixing activity of symbiotic systems of this variant increased by $101 \%$ compared to control 1 in the budding stage and by $80 \%$ in the beans formation stage.

The ferum nanocarboxylate in the biological preparation caused an increase of nitrogen-fixing activity of legume-rhizobial systems in the conditions of optimal water supply relative to control 1 by $17 \%, 106 \%$ and $165 \%$ in the stages of budding, blossom and beans formation, respectively (Table 3). Under the influence of insufficient water supply, plants of this variant were recorded to have increase in nitrogen-fixing activity compared with control 2 by $37 \%, 108 \%$ and $93 \%$ in the stages of budding, blossom and beans formation, respectively.

The use of germanium nanocarboxylate as a component of the inoculation suspension stimulated the nitrogen-fixing activity of root nodules at the optimal growing conditions throughout the vegetation season of soybeans and provided an increase from $63 \%$ to $144 \%$ compared with plants of control variant 1 , starting from the stage of three true leaves to the stage of beans formation. Under the influence of insufficient water supply and the presence of germanium in the content of the inoculant, high indicators of nitrogen-fixing activityof symbiotic systems were observed, and they exceeded the values of control plants (by $30 \%$ of total moisture content) by $23 \%$ in the budding stage, $172 \%$ - in the blossom stage and $116 \%$ - in the beans formation stage.

Under conditions of optimal watering, the beans-rhizobial systems formed as a result of bacterization of soybean seeds with the addition of chromium nanocarboxylate significantly exceeded the control (control 1) according to nitrogen fixation intensity during the whole soybean growing season. In particular, in the stages: three true leaves - by $95 \%$, budding by $83 \%$, blossom - by $135 \%$ and beans formation - by $258 \%$. After the plants of the same variant were kept in the conditions of insufficient water supply (budding stage) for one week, the intensity of nitrogen fixation by them was by $41 \%$ lower compared to control 2 , while the two-week stress did not cause significant changes in the investigated indicator. Resumption of watering to the optimal level in the symbiotic systems formed on the basis of bacterial preparation with the addition of chromium nanocarboxylate caused $43 \%$ increase of nitrogen-fixing activity compared to the control.

In the optimal water supply conditions, cuprum nanocarboxylate increased nitrogen-fixing activityof soybean symbiotic systems formed compared with control plants 1 by $39 \%$ and $142 \%$ in the budding and blossom stages (Table 3). However, in the stage of beans formation, the plants of this variant were characterized by a significant decrease in nitrogen-fixing activity by $54 \%$ compared with the control. The week-long influence of insufficient water supply (budding stage) caused $28 \%$ decrease in nitrogen-fixing activity of symbiotic systems exposed to cuprum compared with control plants 2 . Increasing the duration of the stress factor to two weeks (blossom stage) led to a significant increase (172\%) in the investigated indicator compared to the same control, and an increase in nitrogen-fixing activity by $39 \%$ after resumption of watering to the optimum level (beans formation stage).

In conditions of optimal water supply, the nitrogen-fixing activity of symbiotic systems formed by rhizobia with molybdenum nanocarboxylate was at the level of control 1, except for the beans formation stage, where nitrogen fixation intensity decreased by $42 \%$ compared with the same control. As for the insufficient water supply, the week-long effect of the stress factor caused an insignificant decrease in nitrogen-fixing activity during the budding stage, but in subsequent stages of plant development, the investigated indicator was at the level plants of control 2. 
Table 3

Nitrogen-fixing activity $\left(\mu \mathrm{mol} \mathrm{C}_{2} \mathrm{H}_{4}\right.$ / (plant·h)) of symbiotic systems of soybean-B. japonicum B1-20 under the influence of metalnanocarboxylates against the background of different water supply $(x \pm S E, n=8)$

\begin{tabular}{|c|c|c|c|}
\hline \multirow{2}{*}{ Variants } & \multicolumn{3}{|c|}{ Stages of development in plants } \\
\hline & budding & blossom & beans formation \\
\hline \multicolumn{4}{|c|}{ Optimal water supply ( $60 \%$ of total moisture content) } \\
\hline B. japonicumB1-20 (control 1) & $6.8 \pm 0.3$ & $6.2 \pm 0.8$ & $5.4 \pm 0.6$ \\
\hline cobalt nanocarboxylate & $13.6 \pm 0.6^{* *}$ & $6.5 \pm 0.6$ & $9.7 \pm 0.7^{*}$ \\
\hline ferumnanocarboxylate & $7.9 \pm 0.5$ & $12.8 \pm 1.1^{\times x}$ & $14.3 \pm 1.2^{x \times}$ \\
\hline germaniumnanocarboxylate & $11.9 \pm 1.3^{\# \#}$ & $10.1 \pm 0.9^{\#}$ & $13.2 \pm 0.7^{\#}$ \\
\hline chromiumnanocarboxylate & $12.4 \pm 3.5^{\prime \prime \prime \prime}$ & $14.6 \pm 1.3^{\text {«ा }}$ & 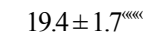 \\
\hline cuprumnanocarboxylate & $9.4 \pm 0.1^{\prime \prime}$ & $16.9 \pm 1.6^{\prime \prime " ' !}$ & $2.5 \pm 0.4^{\prime \prime}$ \\
\hline molybdenumnanocarboxylate & $6.3 \pm 0.2$ & $7.3 \pm 0.4$ & $3.1 \pm 0.9^{+}$ \\
\hline \multicolumn{4}{|c|}{ Insufficient water supply ( $30 \%$ of total moisture content) } \\
\hline cum B1-20 (control 2) & $4.8 \pm 0.1$ & $1.7 \pm 0.2$ & $2.7 \pm 0.3$ \\
\hline cobalt nanocarboxylate & $5.9 \pm 0.2 *$ & $2.6 \pm 0.2 *$ & $6.9 \pm 0.6^{*}$ \\
\hline ferumnanocarboxylate & $6.6 \pm 0.2^{\times}$ & $3.6 \pm 0.6^{\times}$ & $5.2 \pm 1.1^{\times}$ \\
\hline germaniumnanocarboxylate & $5.9 \pm 0.5^{\ddagger}$ & $4.7 \pm 0.5^{\#}$ & $5.8 \pm 0.5^{\ddagger}$ \\
\hline chromiumnanocarboxylate & $2.9 \pm 0.2^{\prime \prime}$ & $1.2 \pm 0.5$ & $3.8 \pm 0.5^{\prime \prime}$ \\
\hline cuprumnanocarboxylate & $3.5 \pm 0.2^{\prime \prime}$ & $4.2 \pm 0.4^{\prime \prime \prime \prime}$ & $3.7 \pm 0.2^{\prime \prime}$ \\
\hline molybdenumnanocarboxylate & $4.4 \pm 0.1$ & $1.9 \pm 0.2$ & $3.3 \pm 0.4^{+}$ \\
\hline
\end{tabular}

Note: see Table 1.

\section{Discussion}

The important indicators of the symbiotic activity of nodule bacteria and legumes are the formation and increase in the weight of active nodules on the roots of plants, which affect the intensity of fixation of molecular nitrogen from the air. According to researchers, the decrease in the intensity of nodulation processes under stress is associated with impaired metabolism in rhizobia cells with insufficient water supply and, as a consequence, leads to decrease in the number of infectious filaments in the root hairs or complete inhibition of their formation.

As a result of our research, a positive effect of inoculation suspension containing cobalt nanocarboxylate on the process of nodules formation in symbiotic systems of soybean-Bradyrhizobium japonicum was revealed at both levels of water supply, optimal and insufficient (Tables 1 and 2). In our opinion, the activating effect of cobalt on the investigated process is associated with its effect on metabolic processes in soybean plants, as the use of cobalt is known to stimulate the formation of chlorophyll. In addition, this element is involved in redox reactions, synthesis of nucleic acids, promotes the intensity of the processes of respiration and synthesis of carbohydrates, fats, vitamins (including ascorbic acid) in the plant organism. It also increases the heat resistance of plants by increasing the synthesis of hydrophilic colloids of the cytoplasm, improving the absorption capacity of roots and increasing the total water content (Chudinova \& Orlova, 2006).

It is important to note that compared with control plants 1 , in the conditions of optimal water supply under the influence of cobalt nanocarboxylate, a larger number nodules formed on soybean roots, but smaller in size. We should also note that researchers have confirmed the ability of cobalt to accumulate in root nodules. This result may be due to an increase in the concentration of this element in the nodules.

Of all the micronutrients, ferum is the one plants need the most. Its content in plants is one hundredth of a percent, but in stressful conditions can increase by several times, and therefore some researchers attribute ferum to macronutrients rather than micronutriebts. This element can enter the plant in the forms of $\mathrm{Fe}^{3+}$ or $\mathrm{Fe}^{2+}$. Dicotyledonous plants mobilize ferum outside the root cells, creating a proton gradient with ATP. Protons increase the solubility of ferum, and electrons reduce $\mathrm{Fe}^{3+}$ to $\mathrm{Fe}^{2+}$, which is transported through the plasmolemma (Kots \& Peterson, 2009).

The stimulating effect of ferum on the formation of the symbiotic apparatus, both in conditions of optimal and insufficient watering is obvious, because it regulates all essential physiological processes of the plant organism (photosynthesis, respiration, protein metabolism, etc.). It is known that ferum is a part of Fe-S clusters, which in turn are components of the cytochrome complex of photosystem I and ferredoxin, and ferredoxin receives electrons from photosystem I and transfers them to $\mathrm{NADP}^{+}$, the lack of this element causes inhibition of photosynthesis (Kots \& Peterson, 2009).

The studies revealed that insufficient water supply did not have such an inhibitory effect on the processes of nodule formation in symbiotic systems influenced by germanium, compared with control plants and during the treatment of seeds with biological preparations with all other nanocarboxylates of metals involved in the study (Tables 1 and 2). According to our data, the week-long effect of insufficient water supply on plants of this variant led to decrease in the mass of root nodules in comparison with plants grown at the optimal water supply. However, the two-week exposure to the stress factor still inhibited the nodulation activity of rhizobia cultivated with germanium and caused decrease in both the number and weight of root nodules compared with plants of the same variant grown at $60 \%$ of complete moisture content. It should be noted that comparison of the indicators of nodulation activity of rhizobia in symbiotic systems of this variant and the indicators of control 2 revealed a protective effect of germanium nanocarboxylate on soybean-rhizobial systems in the conditions of insufficient water supply (Fig. 1). Obviously, this effect of the use of germanium nanocarboxylate is due to its ability to affect the activity of antioxidant enzymes (Menchikov \& Ignatenko, 2012). A team of scientists from Korea conducted a number of studies with Oplopanax elatus plants and confirmed that exogenous germanium is able to increase the antioxidant activity and activity of DPPH and ABTS radicals compared to control plants. In addition, the total content of phenol and flavonoids in experimental plants treated with $50 \mathrm{mg} / \mathrm{L} \mathrm{GeO}_{2}$ was higher than in the control plants (Kim et al., 2016). It is believed that in plants germanium activates the decomposition of water into hydrogen and oxygen, enhances oxygen utilization, and also affects hydrogen ions, alleviates its detrimental effect on the cell, promoting interaction with oxygen by transporting it to all parts of the plant. The mechanism of biological action of germanium compounds is related to the peculiarities of the electronic structure of its atoms that contain 32 electrons, 4 of which are at the external electronic level. When a positively charged ion (or polar molecule) approaches such an atom, one of the outer electrons is easily detached, resulting in the formation of a positively charged ion. In this case, any free electron nearby will aim at filling this niche, and germanium will seek to restore its ordinary electronic structure (Liu et al., 2016).

Chromium is an essential component of plant tissues, but there is very little direct evidence that it is necessary for plant function (Shanker et al., 2009). There are few data that prove the importance of this element for the plant organism. Chromium and a number of other micro- and ultramicroelements were observed to be involved in almost all processes that occur in the plant cell: energy metabolism, primary and secondary metabolism, hormonal regulation, transmitting signals and other. The positive effect of chromium was confirmed for the intensity of photosynthesis in corn plants. We determined that under optimal growing conditions, chromium nanocarboxylate provided one of the highest rates of nodulating activity of rhizobia among all investigated variants. In the conditions of insufficient water supply, this element was also characterized by stimulating effect on the activity of root nodules formation compared with control 2, but the effect of its use was less notable in relation to plants grown under optimal conditions. The positive effect of chromium nanocarboxylate we observed may be related to the valence of this element, as it has been proven that this property can affect the phytotoxicity of metals, for the mobility of the element in soil and its availability to plants depend on it. Hexavalent chromium is an anion of chromic acid and in this form is practically not fixed by soil colloids, as they mostly carry a negative charge, and trivalent chromium acts as a cation, is well fixed in the soil and is low-toxic. It was determined that small amounts of trivalent chromium had a stimulating effect on the formation of root nodules in legumes. Also, the decrease of the negative impact of insufficient water supply caused by the use of chromium may be due to the ability of this element to increase the activities of catalase and proteases. The studies revealed that small concentrations of chromium $(0.05-0.0005 \%)$ stimulate the activity of these enzymes.

Cuprum and molybdenum nanocarboxylates, regardless of the level of water supply, had a less pronounced positive effect on the processes of nodule formation, and in some cases even led to decrease in the investigated indicators compared with control plants (Tables 1 and 2). 
Copper, which is an essential element for plants, in high concentrations can have a toxic effect on them. By its chemical nature, it binds more strongly to organic compounds than to other metals. Copper ions are able to displace the functional metals from enzymes, to interact with biological membranes and reduce molecular oxygen to active oxygen forms. Specifically these properties make copper the strongest toxicant at overdoses. It should be noted that despite the toxic effects of excess copper on living organisms, its lack slows the development of complete nodules on the roots of legumes. Perhaps to obtain a more positive effect on the symbiotic apparatus of soybeans by using this element, it is necessary to correct its concentration.

Molybdenum is an essential microelement for the active functioning of legume-rhizobial symbiosis, which activates the enzymes of symbiotic nitrogen fixation. The available literature data on the effect of this trace element on rhizobia and the subsequent formation of symbiosis are quite contradictory (Rahman et al., 2008).

Thus, the studies found that most of the metal nanocarboxylates we studied activate the nodulation process in soybean-rhizobial systems, and the nature and degree of their impact depend on the element that is being used and the level of water supply. It was found that the least pronounced protective action on the activity of root nodules formation was exhibited by chromium and molybdenum nanocarboxylates. Moreover, under conditions of optimal water supply, chromium nanocarboxylate provided one of the highest indicators of rhizobia nodulation activity. The effect of using cuprum nanocarboxylate varied and depended on both the level of water supply and the stage of plant development. The studies confirmed that the most pronounced protective effect on the symbiotic systems formed under the influence of insufficient water supply was taken by germanium, cobalt and ferum nanocarboxylates. They reduced the negative impact of stress on the investigated process, which is confirmed by the high number and weight of root nodules.

It is known that one of the most important indicators of the effectiveness of the symbiosis of legumes with rhizobia is the nitrogen-fixing activity of nodules. Further analysis of the results of our studies showed that the nitrogen-fixing activity of symbiotic systems that functioned in the conditions of insufficient water supply was significantly lower compared to the activity of similar systems by optimal water supply. At the same time, the use of metal nanocarboxylates mitigated the negative impact of the stress factor on the process of fixation of molecular nitrogen by soybean-rhizobial systems (Table 3 ).

There is a pressumption that mitigation of nitrogen fixation under the influence of drought occurs due to decrease in the amount of carbon entering the bacteroids (Marino et al., 2007). Changes in the activity of sucrose synthase and isocitrate dehydrogenase, as well as malate in root nodules were recorded (Ladrera et al., 2007). Also, the negative effect of insufficient water supply on the nitrogen-fixing activity of the nodules may be related to the accumulation of ureides and asparagine in the nodules (Kurdalai et al., 2002). Sinclair et al. (2003) suggested that differences of ureides metabolism in the leaves were the main reason for the sensitivity of the varieties to drought. These studies were conducted in average-long drought or after exposure to external ureides. However, the use of exogenous ureides to imitate the physiological response to drought demonstrated that these compounds do not reach the nodules (the site of their physiological synthesis) due to xylem. Instead, they accumulate directly in the leaves. King \& Purcell (2005) suggested that the accumulation of ureides in soybean leaves is not the cause of inhibition of nitrogen fixation. These results show that leaf ureides are not involved in the early stages of inhibition of nitrogen fixation during drought, although their role in the later stages of more severe drought cannot be dismissed. At the same time, the same authors noted that the accumulation of ureides in the nodules correlated with the decrease in their nitrogen-fixing activity. Thus, they concluded that increasing the content of these substances in the nodules, along with other nitrogen compounds, may induce inhibition of nitrogen feedback. Ruben et al. (2007) also showed that drought caused the accumulation of ureides in the nodules. However, this effect may have been caused by other factors, such as increased activity of enzymes biosynthesis of ureides. This seems unlikely because the activity of primary metabolism in the nodules is stable, or tends to decrease under mild or moderate drought, respectively. Ruben does not confirm conclusively the fact that ureides are those compounds that cause a decrease in nitrogen-fixing activity when they accumulate in the nodules, but suggests that the accumulation of fixation products is one of the causes of inhibition of nitrogenfixing activity (Ruben et al., 2007). Inhibition of the activity of root nodules due to insufficient water supply may be also associated with lack of energy resources in the plant, which are also spent on maintaining the physiological processes of the plant-macrosymbiont under stress. In addition, inhibition of symbiotic nitrogen fixation may be a consequence of impaired gas exchange in soybean nodules. It was revealed that the decrease in nitrogen-fixing activity under the influence of adverse environmental factors is associated with a decrease in the nodules in the concentration of $\mathrm{O}_{2}$ below optimum level due to sharp decrease in the permeability of the gas diffusion barrier (Gordon et al., 1997; Minchin et al., 2008).

It was found that the positive effect of cobaltnanocarboxylate on the nitrogen fixation intensity of symbiotic systems was more notable in conditions of insufficient water supply and also caused the highest indicators of nitrogen-fixing activity of all experimental variants in the period of resumption of watering (stage of beans formation). In the conditions of optimal water supply of soybeans, the effect of the use of cobaltnanocarboxylate in the composition of the inoculant made on the basis of B. japonicum B1-20 depended on the stage of plant development. Such an effect of cobaltnanocarboxylate on the nitrogen-fixing activity of legume-rhizobial symbiotic systems may be due to its active participation in the synthesis of vitamin $\mathrm{B}_{12}$ and leghemoglobin in the root nodules, and as it is known there is a positive correlation between the nitrogen-fixing activity and the content of these substances. According to B. Yagodin, the nodules of different legumes contain $0.30-1.07 \mu \mathrm{g}$ of cobaltper per $1 \mathrm{~g}$ of dry matter. He also revealed a significant increase in nitrogen-fixing activity in the nodules under the influence of cobalt. Moreover, when solutions of cobalt salts and vitamin $B_{12}$ infiltrated the entire nodules or their sections, the best effect was obtaied by cobalt in ionic form, which was used by rhizobia for biosynthesis of cobalt-containing coenzymes. It can be also assumed that the positive effect of cobalt on the nitrogen fixation intensity of symbiotic systems is associated with its presence in the enzyme methylmalonylisomerase that takes part in one of the stages of conversion of propionate to succinate in root nodules. In addition, this element activates the nitrogenase activity in the nodules, which in turn causes the activation of nitrogen-fixing activity by symbiotic systems (Weisany et al., 2013).

Under the influence of a stress factor lasting two weeks, in plants inoculated with a biological preparation modified of ferumnanocarboxylate, a significant decrease in the intensity of nitrogen fixation was observed compared to plants of the same variant, grown in the conditions of optimal water supply. However, a comparative analysis of nitrogen-fixing activity in plants of this variant with control 2 revealed that Fe nanocarboxylate mitigated the negative effect of insufficient water supply on symbiotic soybean systems and helped to maintain the intensity of molecular nitrogen fixation by root nodules at a fairly high level. Symbiotic systems of this variant quickly restored their activity after resuming the optimal level of water supply, as evidenced by the excess nitrogen fixation intensity, compared with control 2 , in the stage of beans formation. It can be assumed that this result of the use of ferum is associated with its key role in the process of fixing molecular nitrogen. It is known that this element is a component of ferum-containing catalysts' nitrogen-fixing activity: ferredoxin, leghemoglobin, catalase and others and it takes an active part in their synthesis.

The greatest stimulating effect on nitrogen-fixing activityof the symbiotic apparatus of soybean plants grown under conditions of optimal water supply was carried out by chromium nanocarboxylate. In conditions of insufficient water supply, the effect of the use of this element in the composition of the inoculant was less pronounced and depended on the stage of the plant development and the duration of the stress factor (Table 3). It was determined that drought significantly inhibited nitrogen fixation activity by these symbiotic systems in comparison with similar ones formed in the conditions of optimal watering. In this case, compared to control 2, in the blossom stage the nitrogen fixation intensity of the root nodules in plants of this variant did not decrease and the prolonged negative action of the stress factor on the symbiotic systems of control variant 2 was greater than on the symbiotic apparatus of plants exposed to chromium nanocarboxylate, and caused almost a three-fold decrease in the examined indica- 
tor compared to the budding stage. Another evidence that chromium nanocarboxylate in the inoculant is able to mitigate the negative effects of insufficient water supply may be the fact that after a week-long resumption of watering to the optimal water supply (beans formation stage), symbiotic systems formed by soybean plants of the Almaz variety and $B$. japonicum B1-20 actively increased the intensity of molecular nitrogen fixation.

The cuprum nanocarboxylate under conditions of optimal watering provided a fairly high rate of fixation of molecular nitrogen in the stages of budding and blossom. This result is not surprising, because the team of authors confirmed the ability of copper to enhance amino acid synthesis and fixation of molecular nitrogen. In addition, this element is involved in the hormonal regulation of the plant organism and increases its resistance to adverse environmental conditions. It should be noted that in the beans formation stage, plants inoculated with copper nanocarboxylate-containing biological preparation were characterized by significant decrease in nitrogen-fixing activity compared with the control 1 . It is obvious that the decrease in the activity of molecular nitrogen fixation in this stage is the result of aging of bacteroid tissue cells of the nodules, which occurs in the late stage of macrosymbiont development, and cuprum nanocarboxylate, apparently, enhances this process.

Analysis of the nitrogen fixation intensity of the symbiotic apparatus of plants exposed to Mo nanocarboxylate in the inoculant revealed that this element did not have a significant stimulating effect on the nitrogenfixing activity of symbiotic soybean systems, and in some stages of plant development even inhibited the activity of the process compared with the control plants (Table 3). It is known that molybdenum is involved in the fixation of atmospheric nitrogen, affects the stabilization of the structure of nucleic acids, together with ferum performs catalytic and structural functions. Its deficiency leads to a sharp decrease in the content of ascorbic acid in plants, negative changes in nitrogen metabolism (decreased activity of protein synthesis, reduced content of amino acids and amides) (Kostevich \& Asokin, 2008). However, the existing literature data indicate a negative effect of this microelement on rhizobia and the subsequent formation of symbiosis. For example, researchers Albino and Campo shown that molybdenum compounds reduce the survival of bacteria Bradyrhizobium sp. on the surface of the seeds and, accordingly, adversely affect the formation of nodules and the process of nitrogen fixation (Albino \& Campo, 2001).

Also, we should take into account the fact that the elements we used were in the form of nanocarboxylates. As is well known, nanoparticles are being gradually absorbed from the soil, and their ionic forms quickly become involved in the biochemical reactions of living organisms. They are involved in the process of electron transfer by the photosynthetic electronic transport chain in plants, thus intensifying the processes of photosynthesis, increase the activity of enzymes and have a direct effect on the mineral nutrition of plants (Aschberger et al., 2015; Hemraj, 2017). There are preliminary conclusions about the positive effects of nanoparticle-based preparations on the productivity and resistance of plants to adverse environmental factors. It was also found that some nanoparticles exhibit antioxidant enzyme-like ability. They are used to modulate the activity of Photosystem II (PSII) by enhancing water cleavage and oxygen elution, thereby improving electron transfer activity during photophosphorylation (Gogos et al., 2012). Nanoparticles can cause modification of bacterial cell metabolism. Such changes could likely be the reason for the increased nitrogen-fixing activity of root nodules, as nanoparticles are bioactive, i.e. they affect biological objects at the cellular level, increasing the efficiency of biochemical processes in living organisms and participating in development of the balance of micronutrients. Scientists in many studies have already confirmed the ability of nanobiopreparations of biogenic metals to increase the resistance of biological systems to adverse weather conditions (Khot et al., 2012; Scott \& Chen, 2013).

\section{Conclusion}

Thus, the results of our study demonstrate that insufficient water supply caused significant suppression of the formation and functioning of the symbiotic apparatus of soybean plants of both control and all experimental variants. However, the use of most of the metal nanocarboxylates involved as components of bacterial preparations based on B. japonicum B1-20 mitigated the negative impact of insufficient water supply as a stress factor on the studied indicators. At the same time, the most pronounced protective effect was exhibited by the nanocarboxylates germanium, cobalt and ferum, which regulated the nodulation activity of nodule bacteria and provided maximum fixation of molecular nitrogen by symbiotic soybean systems in conditions of insufficient water supply, and stimulated active recovery after exposure to stressors. The obtained data suggest that the use of germanium, cobalt and ferum nanocarboxylates in the concentration of 1:1,000 in microbial preparations based on active strains of nodule bacteria promotes the adaptation of the formed legume-rhizobial symbiotic systems to drought and can be a significant tool for increasing their resistance to insufficient water supply.

\section{References}

Albino, U. B., \& Campo, R. J. (2001). Effect of sources and levels of molybdenum on Bradyrhizobium survival and on biological nitrogen fixation in soybean. Pesquisa Agropecuária Brasileira, 36(3), 527-534.

Aldasoro, J., Larrainzar, E., \& Arrese-Igor, C. (2019). Application of anti-transpirants temporarily alleviates the inhibition of symbiotic nitrogen fixation in droughtstressed pea plants. Agricultural Water Management, 213, 193-199.

Aschberger, K., Gottardo, S., Amenta,V., Arena, M., Botelho Moniz, F., Bouwmeester, H., Brandhoff, P., Mech., A., Quiros Pesudo, L., \& Rauscher, H. (2015). Nanomaterials in food - current and future applications and regulatory aspects. Journal of Physics: Conference Series, 617, 012032.

Chudinova, L. A., \& Orlova, N. V. (2006). Fiziologiya ustoychivosti rasteniy [Physiology of plant resistance]. Perm University Printing House, Perm (in Russian).

Dimkpa, C. O., Bindraban, P. S., Fugice, J., Agyin-Birikoran, S., Singh, U., \& Hellum, D. (2017). Composite micronutrient nanoparticles and salts decrease drought stress in soybean. Agronomy for Sustainable Development, $37,5$.

Du, W., Tan, W., Peralta-Videa, J. R., Gardea-Torresdey, J. L., Ji, R., Yin, Y., \& Guo, H. (2017). Interaction of metal oxide nanoparticles with higher terrestrial plants: Physiological and biochemical aspects. Plant Physiology and Biochemistry, 110, 210-225.

Gogos, A., Knauer, K., \& Bucheli, T. D. (2012). Nanomaterials in plant protection and fertilization: Current state, foreseen applications, and research priorities. Journal of Agricultural and Food Chemistry, 60, 9781-9792.

Gordon, A. J., Minchin, F. R., Skot, L., \& James, C. L. (1997). Stress-induced declines in soybean $\mathrm{N}_{2}$ fixation are related to nodule sucrose synthase activity. Plant Physiology, 114(3), 937-946.

Hemraj, C. (2017). Nanopesticide: Current status and future possibilities. Agricultural Research and Technology, 5(1), 555651.

Javaid, M., Naeem-Ullah, U., Khan, W. S., Saeed, S., Qayyum, M. A., \& Khan, M. A. (2020). Role of nanotechnology in crop protection and production: A review. Journal of Innovative Sciences, 6(2), 221-227.

Khot, L. R., Sankaran, S., Maja, J. M., Ehsani, R., \& Schuster, E. W. (2012). Applications of nanomaterials in agricultural production and crop protection: A review. Crop Protection, 35, 64-70.

Kibido, T., Kunert, K., Makgopa, M., Greve, M., \& Vorster, J. (2019). Improvement of rhizobium-soybean symbiosis and nitrogen fixation under drought. Food and Energy Security, 9, e177.

Kim, H. Y., Seong, E. S., Yoo, J. H., Choi, J. H., Kang, B. J., Jeon, M. R., Kim, M. J., \& Yu, C. Y. (2016). Effect of germanium treatment on growth and production of organic germanium in Oplopanax elatus. Название журнала, 24(3), 214-221.

King, C. A., \& Purcell, L. C. (2005). Inhibition of $\mathrm{N}_{2}$ fixation in soybean is associated with elevated ureides and amino acids. Plant Physiology, 137(4), 1389-1396.

Kostevich, S. V., \& Asokin, O. I. (2008). Primenenie bora i molibdena na posevakh soi [The use of boron and molybdenum in soybean crops]. Nauchno-tekhnicheskiy Byulleten Vserossiyskogo Nauchno-Issledovatel'skogo Instituta Maslichnykh Kul'tur, 139, 1-5 (in Russian).

Kots, S. Y., \& Peterson, N. V. (2009). Mineral'ni elementy i dobryva v zhyvlenni roslyn [Mineral elements and fertilizers in plant nutrition]. Logos, Kyiv (in Ukrainian).

Kunert, K. J., Vorster, B. J., Fenta, B. A., Kibido, T., Dionisio, G., \& Foyer, C. H. (2016). Drought stress responses in soybean roots and nodules. Frontiers in Plant Science, 7, 1015.

Kurdalai, F., Al-Ain, F., \& Al-Shamm, A. M. (2002). Nodulation, dry matter production, and $\mathrm{N}_{2}$ fixation by faba bean and chickpea as affected by soil moisture and potassium fertilizer. Journal of Plant Nutrition, 25(2), 355-368.

Ladrera, R., Marino, D., Larrainzar, S., González, E. M., \& Arrese-Igor, C. (2007). Reduced carbon availability to bacteroids and elevated ureides in nodules, but not in shoots, are involved in the nitrogen fixation response to early drought in soybean. Plant Physiology, 145(2), 539-546.

Liu, Y., Hou, L., Li, Q., Jiang, Z., Liu, D., \& Zhu, Y. (2016). The effects of exogenous antioxidant germanium $(\mathrm{Ge})$ on seed germination and growth of Lycium 
ruthenicum Murr subjected to $\mathrm{NaCl}$ stress. Environmental Technology, 37(8), 909-919.

Marino, D., Frendo, P., Ladrera, R., Zabalza, A., Puppo, A., Arrese-Igor, C., \& González, E. M. (2007). Nitrogen fixation control under drought stress. Localized or systemic? Plant Physiology, 143(4), 1968-1974.

Menchikov, L. G., \& Ignatenko, M. A. (2012). Biologicheskaya aktivnost' organicheskikh soedineniy germaniya (obzor) [Biological activity of organogermanium compounds (a review)]. Pharmaceutical Chemistry Journal, 46(11), 3-6 (in Russian).

Minchin, F. R., James, E. K., \& Becana, M. (2008). Chapter 7. Oxygen diffusion, production of reactive oxygen and nitrogen species, and antioxidants in legume nodules. In: Редактора Nitrogen-fixing leguminous symbioses. Издательство, города. Рp. 321-362.

Parvaze, A. S., Baba, Z. A., Hamid, B., \& Swaroop, R. M. (2018). Harnessing soil rhizobacteria for improving drought resilience in legumes. In: Редактора Legumes for soil health and sustainable management. Издательство, города. Рр. 235-275.

Patra, J. K., \& Baek, K.-H. (2017). Antibacterial activity and synergistic antibacterial potential of biosynthesized silver nanoparticles against foodbome pathogenic bacteria along with its anticandidal and antioxidant effects. Frontiers in Microbiology, $8,167$.

Prasad, R., Bhattacharyya, A., \& Nguyen, Q. D. (2017). Nanotechnology in sustainable agriculture: Recent developments, challenges, and perspectives. Frontiers in Microbiology, 8, 1014.

Rahimi, D., Kartoolinejad, D., Nourmohammadi, K., \& Naghdi, R. (2016). Increasing drought resistance of Alnus subcordata C. A. Mey. seeds using a nano priming technique with multi-walled carbon nanotubes. Journal of Forest Science, 62(6), 269-278.

Rahman, M. M., Bhuiyan, M. M. H., Sutradhar, G. N. C., Rahman, M. M., \& Paul, A. K. (2008). Effect of phosphorus, molybdenum and rhizobium inoculation on yield and yield attributes of mungbean. International Journal of Sustainable Crop Production, 3(6), 26-33.
Ruben, L., Marino, D., Larrainzar, S., González, E. M., \& Arrese-Igor, C. (2007). Reduced carbon availability to bacteroids and elevated ureides in nodules, but not in shoots, are involved in the nitrogen fixation response to early drought in soybean. Plant Physiology, 145(2), 539-546.

Schwember, A. R., Schulze, J., Pozo, A., \& Cabeza, R. A. (2019). Regulation of symbiotic nitrogen fixation in legume root nodules. Plants, 8(9), 333.

Scott, N., \& Chen, H. (2013). Nanoscale science and engineering for agriculture and food systems. Industrial Biotechnology, 9(1), 17-18.

Shanker, A. K., Djanaguiraman, M., \& Venkateswarlu, B. (2009). Chromium interactions in plants: Current status and future strategies. Metallomics, 1(5), 375-383.

Sinclair, T. R., Vadez, V., \& Chenu, K. (2003). Ureide accumulation in response to Mn nutrition by eight soybean genotypes with $\mathrm{N}_{2}$ fixation tolerance to soil drying. Crop Science, 43(2), 592-597.

Solanki, P., Bhargava, A., Chhipa, H., Jain, N., \& Panwar, J. (2015). Nano-fertilizers and their smart delivery system. In: Rai, M., Ribeiro, C., Mattoso, L., \& Duran, N. (Eds.). Nanotechnologies in Food and Agriculture. Springer International Publishing. Pp. 81-101.

Sonali, R., Liu, W., Nandet, R. S., Crook, A., Mysore, K. S., Pislariu, C. I., Frugoli, J., Dickstein, R., \& Udvardi, M. K. (2020). Celebrating 20 years of genetic discoveries in legume nodulation and symbiotic nitrogen fixation. The Plant Cell, 32(1), 15-41.

Wang, Q., Liu, J., \& Zhu, H. (2018). Genetic and molecular mechanisms underlying symbiotic specificity in legume-rhizobium interactions. Frontiers in Plant Science.

Weisany, W., Raei, Y., \& Allahverdipoor, K. H. (2013). Role of some of mineral nutrients in biological nitrogen fixation. Bulletin of Environment, Pharmacology and Life Sciences, 2(4), 77-84.

Worrall, V. S., \& Roughley, R. J. (1976). The effect of moisture stress on infection of Trifolium subterraneum L. by Rhizobium trifolii Dang. Journal of Experimental Botany, 27, 1233-1241.

Zahran, H. H., \& Sprent, J. I. (1986). Effects of sodium chloride and polyethylene glycol on root hair infection and nodulation of Vicia faba L. plants by Rhizobium leguminosarum. Planta, 167(3), 303-309. 